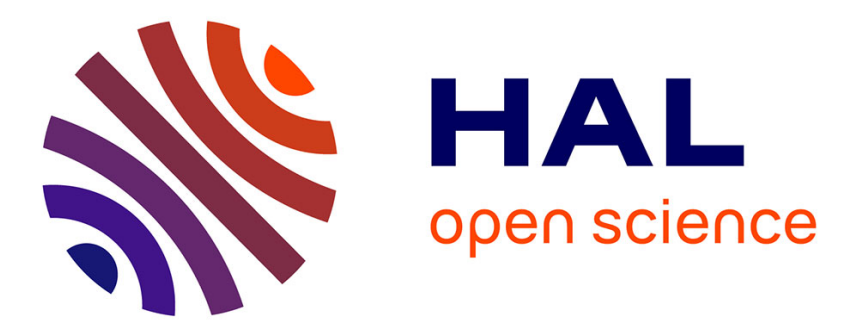

\title{
Modelling honeybee visual guidance in a 3-D environment
}

G. Portelli, Julien Serres, F. Ruffier, N. Franceschini

\section{To cite this version:}

G. Portelli, Julien Serres, F. Ruffier, N. Franceschini. Modelling honeybee visual guidance in a 3-D environment. Journal of Physiology - Paris, 2010, 104 (1-2), pp.27-39. 10.1016/j.jphysparis.2009.11.011 . hal-02010550

\section{HAL Id: hal-02010550 https://hal-amu.archives-ouvertes.fr/hal-02010550}

Submitted on 7 Feb 2019

HAL is a multi-disciplinary open access archive for the deposit and dissemination of scientific research documents, whether they are published or not. The documents may come from teaching and research institutions in France or abroad, or from public or private research centers.
L'archive ouverte pluridisciplinaire $\mathbf{H A L}$, est destinée au dépôt et à la diffusion de documents scientifiques de niveau recherche, publiés ou non, émanant des établissements d'enseignement et de recherche français ou étrangers, des laboratoires publics ou privés. 


\title{
Modelling honeybee visual guidance in a 3-D environment
}

\author{
Portelli G., Serres J., Ruffier F., and Franceschini N. \\ The Institute of Movement Sciences, UMR6233 CNRS - Aix-Marseille Uni., CP938, 163 \\ ave. Luminy, 13288 Marseille Cedex 09, France
}

\begin{abstract}
In view of the behavioral findings published on bees during the last two decades, it was proposed to decipher the principles underlying bees' autopilot system, focusing in particular on these insects' use of the optic flow (OF). Based on computer-simulated experiments, we developed a vision-based autopilot that enables a "simulated bee" to travel along a tunnel, controlling both its speed and its clearance from the right wall, left wall, ground, and roof. The flying agent thus equipped enjoys three translational degrees of freedom on the surge $(x)$, sway $(y)$, and heave $(z)$ axes, which are uncoupled. This visuo-motor control system, which is called ALIS (AutopiLot using an Insect based vision System), is a dual OF regulator consisting of two interdependent feedback loops, each of which has its own OF set-point. The experiments presented here showed that the simulated bee was able to navigate safely along a straight or tapered tunnel and to react appropriately to any untoward OF perturbations, such as those resulting from the occasional lack of texture on one wall or the tapering of the tunnel. The minimalistic visual system used here (involving only eight pixels) suffices to jointly control both the clearance from the four walls and the forward speed without
\end{abstract}


having to measure any speeds or distances. The OF sensors and the simple visuo-motor control system we have developed account well for the results of ethological studies performed on honeybees flying freely along straight and tapered corridors.

Key words: Optic Flow (OF), computational neurosciences, honeybee, speed control, biomimetics, obstacle avoidance. 


\section{INTRODUCTION}

2

Winged insects are able to navigate in unfamiliar environments, using the optic flow (OF) (Gibson, 1950) generated by their own motion (Horridge, 1987). Insects make use of the OF to avoid lateral obstacles (Srinivasan et al., 1991; Serres et al., 2008b), control their speed (Preiss, 1987; Baird et al., 2005, 2006) and height (Baird et al., 2006; Franceschini et al., 2007), cruise and land (Srinivasan et al., 1996, 2000; Franceschini et al., 2007). Behavioral studies on flying insects have inspired several researchers to develop visually guided mobile robots (Pichon et al., 1989; Franceschini et al., 1992; Coombs and Roberts, 1992; Duchon and Warren, 1994; Santos-Victor et al., 1995; Weber et al., 1997; Lewis, 1997; Netter and Franceschini, 2002; Ruffier and Franceschini, 2003; Humbert et al., 2007; Beyeler et al., 2007).

The LORA III autopilot we previously developed was based on a pair of lateral OF regulators steering a fully actuated hovercraft, in which the surge and sway dynamics were uncoupled (Serres et al., 2008a). The LORA III autopilot was found to account for the behaviors such as centering and speed control observed in bees flying along stationary and nonstationary corridors (Srinivasan et al., 1991) as well as tapered corridors (Srinivasan et al., 1996). LORA III also accounted for the novel findings on wall-following (Serres et al., 2008b), which the previous hypothesis ("optic flow balance" hypothesis) could not explain.

In the ALIS autopilot described here, the LORA III autopilot principle is extended to include the vertical plane. The problem consisted here of developing a functional scheme for a joint speed control and obstacle avoidance system that would take not only lateral obstacles but also ventral obstacles 
(Baird et al., 2006; Franceschini et al., 2007), and dorsal obstacles (Vickers and Baker, 1994) into account. The ALIS autopilot we designed was used to test a simulated honeybee, in which all the translational degrees of freedom (DOF) (surge, sway, and heave) were uncoupled (Ellington, 1984). In our simulations, the flying agent was endowed with the following novel flight features:

- use of 2-D model for photoreceptor sensitivity,

- use of the walls, ground, and roof, which were all textured with natural scenes,

- use of a new linearized model for flying bees' locomotion.

- use of an optic flow regulator based on both the lateral and the vertical OFs.

The ALIS autopilot regulates the OF thanks to the positioning and forward control systems with which it is equipped, according to the following principles:

(i) the first OF regulator adjusts the bee's forward speed so as to keep whichever sum of the two opposite OFs (i.e., left/right or ventral/dorsal) is maximum equal to a forward OF set-point. The outcome is that the bee's forward speed becomes proportional to the smallest dimension (either the width or the height) of the flight tunnel. The forward speed attained will be such that the OF generated equals the value of the forward OF set-point.

(ii) the second OF regulator adjusts the bee's lateral or vertical position so as to keep whichever OF is maximum (among the four OFs : left, right, 
ventral, and dorsal) equal to the positioning OF set-point. The outcome is that the clearance from the nearest tunnel surface (the walls, ground, or roof) becomes proportional to the bee's current forward speed, as defined in (i). The clearance from the nearest tunnel will be such that the OF generated equals to the positioning OF set-point.

The ALIS autopilot enables the agent to perform obstacle avoidance by performing maneuvers involving only translational DOFs, unlike the obstacle avoidance schemes based on body saccades that involve rotational DOFs (Lewis, 1997; Schilstra and van Hateren, 1999; Tammero and Dickinson, 2002;

Beyeler et al., 2007). The ALIS autopilot operates without relying on any speed or distance measurements. It also differs fundamentally from previous "insect-like" navigation systems based on speed or distance regulation (Dickson et al., 2006).

In section 2 , the dynamical model for the simulated bee is described in terms of its three translational DOFs. In section 3, the simulation set-up used to test the ALIS autopilot on board the simulated bee is described. Section 4 describes the ALIS autopilot in detail. Section 5 gives the results of computer-simulated experiments carried out on the simulated bee, which is able to perform various tasks such as takeoff, straight and tapered tunnelfollowing, and to react appropriately to any local lack of lateral or dorsal $\mathrm{OF}$.

\section{DYNAMICAL MODEL FOR BEES' FLIGHT}

Here we focus on the visuomotor feedback loops that may explain how a flying insect controls its speed and avoids obstacles. A linearized model 
for the bee's dynamics is proposed in terms of the three translational DOFs (surge, sway, and heave dynamics). Linearization was justified here by the limited range of speeds $(0-2 \mathrm{~m} / \mathrm{sec})$ possible. The value of the three rotational DOFs was kept at zero because bees are known to fly straight to their nectar source (von Frisch, 1948; Riley et al., 2003). In our experiments, the simulated insect was not subjected to any wind disturbances: the groundspeed was therefore taken to be equal to the airspeed. The bee's dynamic performances in the three translational DOFs will be described in detail below.

\subsection{Bees' Surge dynamics}

(FIGURE 1 about here)

Experiments on fruitflies (David, 1978) and honeybees (Nachtigall et al., 1971; Esch et al., 1975) have shown that flying insects gain forward speed by pitching their mean flight-force vector $\vec{F}$ forward at a small angle $\theta_{\text {pitch }}$ ( $\leq 20 \mathrm{deg}$ ) with respect to the vertical (Fig. 1A, B). By slightly changing the wing stroke plane pitch angle $\theta_{\text {pitch }}$, the insect generates a forward Thrust $T$, which hardly affects the vertical Lift $L$ (Ellington, 1984). In bees, the mean flight-force vector orientation differs from the body orientation, forming a fixed angle (Nachtigall et al., 1971; Ellington, 1984).

\subsection{Bees' Sway dynamics}

In flying hymenopterans, sideslip motion results from roll changes (Ellington, 1984; Zeil et al., 2008). The wing stroke plane roll angle $\theta_{\text {roll }}$ therefore drives the Sideways thrust $S$ (Fig. 1A, C). 


\subsection{Bees' Heave dynamics}

The mean flight-force vector $\vec{F}$ (Eq. 1)resulting from the wing stroke amplitude $\Phi$ (Dillon and Dudley, 2004; Altshuler et al., 2005) can be expressed in terms of forward Thrust $T$, Side thrust $S$, and vertical Lift $L$.

$$
\vec{F}=\left\{\begin{array}{c}
T \\
S \\
L
\end{array}\right\}=\left\{\begin{array}{l}
F(\Phi) \cdot \sin \theta_{\text {pitch }} \cdot \cos \theta_{\text {roll }} \\
F(\Phi) \cdot \cos \theta_{\text {pitch }} \cdot \sin \theta_{\text {roll }} \\
F(\Phi) \cdot \cos \theta_{\text {pitch }} \cdot \cos \theta_{\text {roll }}
\end{array}\right\}
$$

where $F(\Phi)$ is the force generated by an amplitude $\Phi$ of the wing stroke. At small angles $\left(\theta_{\text {pitch }}\right.$ and $\left.\theta_{\text {roll }}\right)$ angles, $L$ is roughly equal to $F$. The wing stroke amplitude $\Phi$ therefore mainly drives the vertical lift $L$.

\subsection{Calculating the gain between the wing stroke amplitude and the lift}

The lift produced by a bee depends on both the density $\rho$ of the air and the wing stroke amplitude $\Phi$ (Dudley, 1995). In order to determine the gain $K_{\text {wing }}$ between the wing stroke amplitude $\Phi$ and the lift $L$, we used the results of experiments on hovering bees that were carried out in media with different densities. Hovering bees were filmed in normal air $\left(\rho_{\text {Air }}=1,21 \mathrm{~kg} / \mathrm{m}^{3}\right)$ and in heliox $\left(\rho_{\text {Heliox }}=0,41 \mathrm{~kg} / \mathrm{m}^{3}\right)$ (Altshuler et al., 2005). In the low density heliox, bees were found to increase their wing stroke amplitude $\Phi$ from $90 \mathrm{deg}$ to 130 deg, while keeping their wingbeat frequency constant. In these two hovering situations $\left(\theta_{\text {pitch }}=\theta_{\text {roll }}=0^{\circ}\right)$, the lift $L$ is equal to the weight:

$$
L_{\text {Heliox }}(\Phi=130 \mathrm{deg})=L_{\text {Air }}(\Phi=90 \mathrm{deg})=m \cdot g \cong 1 \mathrm{mN}
$$

In a steady state analysis, the lift is proportional to the density at a given stroke amplitude $\Phi=130 \mathrm{deg}$ (Ellington, 1984; Sane and Dickinson, 2002): 


$$
L_{\text {Air }}(\Phi=130 \mathrm{deg}) / \rho_{\text {Air }}=L_{\text {Heliox }}(\Phi=130 \mathrm{deg}) / \rho_{\text {Heliox }}
$$

115

We therefore calculated $L_{A i r}(\Phi=130 \mathrm{deg}) \cong 3 \mathrm{mN}$, and obtained the mean sensitivity of the lift production to the wing stroke amplitude, $K_{\text {wing }}=$ $\partial L_{\text {Air }} / \partial \Phi=50 \mu \mathrm{N} / \mathrm{deg}$ in hovering bees $\left(\Phi_{\text {Hover }}=90 \mathrm{deg}\right)$.

\subsection{The Linearized Flying Bee model}

At small pitch levels $\left|\theta_{\text {pitch }}\right| \leq 20$ deg and roll $\left|\theta_{\text {roll }}\right| \leq 20$ deg angles, each component of the mean flight-force vector $\vec{F}$ can be linearized on the surge, sway, and heave axes (Eq. 2) as a function of the pitch angle $\theta_{\text {pitch }}$, the roll angle $\theta_{\text {roll }}$, and the wing stroke amplitude $\Phi=\Phi_{\text {Hover }}+\Delta \Phi$, respectively:

$$
\vec{F}=\left\{\begin{array}{c}
T \\
S \\
L
\end{array}\right\}=\left\{\begin{array}{c}
m \cdot g \cdot \theta_{\text {pitch }} \\
m \cdot g \cdot \theta_{\text {roll }} \\
K_{\text {wing }} \cdot\left(\Phi_{\text {Hover }}+\Delta \Phi\right)
\end{array} \quad \text { with } K_{\text {wing }} \cdot \Phi_{H o v e r}=m \cdot g\right.
$$

The following linearized system of equations was referred to the bee's center of gravity as follows:

$$
m \cdot d \vec{V} / d t+Z \cdot \vec{V}=\vec{F}+m \cdot \vec{g}
$$

${ }_{127}$ viscous friction matrix $Z=\left[\begin{array}{ccc}\zeta & 0 & 0 \\ 0 & \zeta & 0 \\ 0 & 0 & \zeta\end{array}\right]$. 
The time constant along a translational DOF can be defined by the ratio between the mass and the translational viscous friction coefficient. To the best of our knowledge, no data are available so far on the sway and heave time constants in the case of freely flying honeybees but these values are likely to be of the same order as the surge time constant. The bee's surge time constant $\tau=m / \zeta=0.22 \mathrm{sec}$ can be estimated from bees' landing data (Srinivasan et al., 2000) and from bees OF based autopilot system (Franceschini et al., 2007). In what follows, bee sway and bee heave time constants are assumed to be equal to the bee surge time constant.

Equation 3 can be written as follows:

$$
\left\{\begin{array}{l}
\tau \cdot d V_{x} / d t+V_{x}=(m \cdot g) / \zeta \cdot \theta_{\text {pitch }} \\
\tau \cdot d V_{y} / d t+V_{y}=(m \cdot g) / \zeta \cdot \theta_{\text {roll }} \\
\tau \cdot d V_{z} / d t+V_{z}=\left(K_{\text {wing }} / \zeta\right) \cdot \Delta \Phi
\end{array}\right.
$$

The sensitivity $K_{\text {surge }}$ of the forward speed $V_{x}$ to the pitch angle $\theta_{\text {pitch }}$ can be determined from figure $2 \mathrm{~b}$ in Esch et al. (1975) and estimated as follows:

$$
K_{\text {surge }}=\left|\partial V_{x} / \partial \theta_{\text {pitch }}\right|=0.10 \mathrm{~m} \cdot \mathrm{sec}^{-1} \cdot \mathrm{deg}^{-1}
$$

$K_{\text {sway }}$ is assumed to have a similar value: $K_{\text {sway }}=K_{\text {surge }}$

The Laplace transfer functions giving the bee's surge dynamics $G_{V_{x}}(s)$, sway dynamics $G_{V_{y}}(s)$, and heave dynamics $G_{V_{z}}(s)$ can therefore be written as follows: 


$$
\left\{\begin{array}{l}
G_{V_{x}}(s)=\frac{V_{x}(s)}{\theta_{\text {pitch }}(s)}=\frac{K_{\text {surge }}}{1+\tau_{\text {surge }} \cdot s}=\frac{0.10}{1+0.22 \cdot s} \\
G_{V_{y}}(s)=\frac{V_{y}(s)}{\theta_{\text {roll }}(s)}=\frac{K_{\text {sway }}}{1+\tau_{\text {sway }} \cdot s}=\frac{0.10}{1+0.22 \cdot s} \\
G_{V_{z}}(s)=\frac{V_{z}(s)}{\Delta \Phi(s)}=\frac{K_{\text {wing }} / \zeta_{z}}{1+\tau_{\text {heave }} \cdot s}=\frac{0.11}{1+0.22 \cdot s}
\end{array}\right.
$$

The pitch angle was limited here to $\left|\theta_{\text {pitch }}\right| \leq 20 \mathrm{deg}$ so as to keep the maximum forward speed range to $V_{x M a x}=2 \mathrm{~m} / \mathrm{sec}$, and the roll angle was limited to $\left|\theta_{\text {roll }}\right| \leq 5 \mathrm{deg}$ so as to keep the maximum lateral speed range to $V_{y \text { Max }}=0.5 \mathrm{~m} / \mathrm{sec}$. Bees are thought to reach the maximum stroke amplitude $\Phi_{\max }=140 \mathrm{deg}$ and the minimum stroke amplitude $\Phi_{\min }=$ 70 deg (Dudley, 2000; Dillon and Dudley, 2004). The maximum ascent speed $V_{z U p M a x}$ and the maximum descent speed $V_{z \text { DownMax }}$ on the heave-axis are therefore:

$$
\left\{\begin{array}{l}
V_{z \text { UpMax }}= \\
\quad\left(K_{\text {wing }} / \zeta_{z}\right) \cdot\left(\Phi_{\text {max }}-\Phi_{\text {Hover }}\right)=5.5 \mathrm{~m} / \mathrm{sec} \\
V_{z \text { DownMax }}= \\
\quad\left(K_{\text {wing }} / \zeta_{z}\right) \cdot\left(\Phi_{\text {Hover }}-\Phi_{\text {min }}\right)=-2.2 \mathrm{~m} / \mathrm{sec}
\end{array}\right.
$$

The bees' ascent speed, was calculated from figure $7 \mathrm{~b}$ in Srinivasan et al. (2000) and found to be equal to $\approx 2 \mathrm{~m} / \mathrm{sec}$. The bees' descent speed measured during landing manoeuvers reaches a value of $2 \mathrm{~m} / \mathrm{sec}$ (figure $6 \mathrm{~d}$ in Srinivasan et al. (2000)): this value is quite similar to our own predictions (Eq. 6). In order to limit the vertical speed $\left(\left|V_{z}\right|=2 \mathrm{~m} / \mathrm{sec}\right)$, we set the maximum stroke amplitude at $|\Delta \Phi| \leq 18$ deg. 


\section{SIMULATION SET-UP}

\subsection{Simulated 3-D environment}

The simulated 3-D visual environment consisted of a straight or tapered flight tunnel (6 meters long, 1 meter wide, and 1 meter high), the four walls of which were lined with high resolution photographs of natural panoramic scenes (Brinkworth and O'Carroll, 2007). These images were converted into 256 grayscale levels and resized keeping the original size ratios. One image pixel corresponded to one millimeter of the simulated environment (Fig. 2). The four natural grayscale images are shown in Fig. 2: right wall (Fig. 2A), left wall (Fig. 2B), roof (Fig. 2C), and ground (Fig. 2D).

\section{(FIGURE 2 about here)}

\subsection{Optic flow generated by the bee's own motion}

The simulated bee was assumed to be flying at a speed vector $\vec{V}$ along the flight tunnel covered with natural-scene textures (Fig. 2). It has been shown that hymenopterans stabilize their gaze by compensating for any body rotations (Zeil et al., 2008), in much the same way as the blowfly does (Schilstra and van Hateren, 1999). The bee's head orientation was therefore assumed to be locked to the $\mathrm{X}$-axis of the tunnel. Since any rotation is compensated for, each OF sensor will receive a purely translational OF, which is the angular velocity of the environmental features detected by the lateral (diametrically opposed) and vertical (also diametrically opposed) OF sensors (Fig. 3).

The translational OF can be defined simply as the forward speed-todistance ratio (expressed in $\mathrm{rad} / \mathrm{sec}$ ) in line with (7). 


$$
\omega_{i}=V_{x} / D_{i}, \quad \text { with } \quad i \in\{R g h t, \text { Left, Vtrl,Drsl }\}
$$

where $V_{x}$ is the bee's forward speed, $D_{R g h t}, D_{\text {Left }}$ are the distances to the side (right and left) walls, and $D_{V t r l}, D_{D r s l}$ are the distances to the ground (ventral eye) and to the roof (dorsal eye) (Fig. 3). Each OF sensor receives its own OF, which can be a right OF $\left(\omega_{R g h t}\right)$, a left OF $\left(\omega_{\text {Left }}\right)$, a ventral OF $\left(\omega_{V t r l}\right)$, or a dorsal OF $\left(\omega_{D r s l}\right)$.

\section{(FIGURE 3 about here)}

\subsection{OF sensors on board the simulated bee}

Bees are endowed with two compound eyes, each of which is composed of 4500 ommatidia. The visual axes of two adjacent ommatidia are separated by an interommatidial angle $\Delta \varphi$, which varies from one region of the eye to another (Seidl and Kaiser, 1981). Each ommatidium is composed of a lens and nine photoreceptor cells with identical receptive fields. Six of these cells have a green spectral sensitivity (Wakakuwa et al., 2005) and are involved in motion vision. These photoreceptor cells are connected to three successive visual optic lobes: the lamina, the medulla, and the lobula. Further down the visual processing chain, descending neurons have been found to respond to object velocity (Velocity-Tuned motion-sensitive neurons VT cells in Ibbotson (2001)). VT neurons respond monotonically to front-to-back translational movements, and therefore act like real OF sensors. Our simulated bee is equipped with only four OF sensors (two lateral, one ventral, and one dorsal sensor, Fig. 3A). Each of these sensors consists of only two photoreceptors (two pixels) driving an Elementary Motion Detector (EMD). 
The visual axes of the two photoreceptors are assumed to be separated by an angle $\Delta \varphi=4$ deg. Each photoreceptor's angular sensitivity is assumed to be a Gaussoid function with an acceptance angle (angular width at half height) $\Delta \rho=4 \mathrm{deg}$, and a total field of view of $10.4 \mathrm{deg} \times 10.4 \mathrm{deg}$. The photoreceptors' output was computed at each time step $(0.5 \mathrm{msec})$ by multiplying two matrixes:

- a matrix representing the visible local natural scene (Fig. 2),

- a matrix representing the insect-like photoreceptor Gaussoid sensitivity.

The "time-of-travel" scheme of the bio-inspired EMD developed by Franceschini's research group has been previously described in detail (Blanes, 1986; Pudas et al., 2007; Aubépart and Franceschini, 2007; Franceschini et al., 2009). The response of this OF sensor is a monotonic function of the angular velocity within a 10-fold range (from $40 \mathrm{deg} / \mathrm{sec}$ to $400 \mathrm{deg} / \mathrm{sec}$ ) (Ruffier and Franceschini, 2005), resembling that of the Velocity-Tuned motion-sensitive descending neurons found to exist in honeybees(VT neurons: Ibbotson, 2001).

\section{THE ALIS AUTOPILOT}

The simulated bee is controlled by an autopilot called ALIS (which stands

for AutopiLot using an Insect-based vision System), which is reminiscent of both the OCTAVE autopilot for ground avoidance (Ruffier and Franceschini, 2005) and the LORA III autopilot for speed control and lateral obstacle avoidance (Serres et al., 2008a) previously developed at our laboratory. The ALIS autopilot relies, however, on four OF measurements: right, left, ventral, and dorsal. We designed the ALIS autopilot assuming that speed control 
and obstacle avoidance problems could be solved in a similar way in both the horizontal and vertical planes. The ALIS autopilot consists of two visuomotor feedback loops: the speed control loop (on the surge axis) and the positioning control loop (on the sway and heave axes). These two loops work in parallel and are interdependent. Each of them involves multiple processing stages (Fig. 4), and each has its own OF set-point: the forward OF set-point and the positioning OF set-point, respectively. In this dual control system, neither the speed nor the distance from the tunnel surfaces (walls, ground, or roof) need to be measured. The simulated bee will react to any changes in the OFs by selectively adjusting the three orthogonal components $V_{x}, V_{y}$, and $V_{z}$ of its speed vector $\vec{V}$.

\section{(FIGURE 4about here)}

\subsection{Forward speed control and forward speed criterion}

The speed control loop was designed to hold the maximum sum of the two diametrically opposed OFs (measured in the horizontal and vertical planes) constant and equal to a forward OF set-point $\omega_{\text {set } F w d}$. The ALIS autopilot does so by adjusting the forward thrust $T$ (that will determine the forward speed $V_{x}$ ). In other words, this regulation process consists in first determining whether the sum of the OFs measured in the horizontal plane $\left(\omega_{R g h t}^{m}+\omega_{\text {Left }}^{m}\right)$ or the sum of those measured in the vertical plane $\left(\omega_{V t r l}^{m}+\omega_{D r s l}^{m}\right)$, is the larger of the two. The larger of the two sums is then compared with the forward OF set-point $\omega_{\text {setFwd }}$ (blue loop, Fig. 4). The forward OF set-point was set at: $\omega_{\text {setFwd }}=4.57 \mathrm{~V}$ (i.e., $540 \mathrm{deg} / \mathrm{sec}$ ). This value was based on that recorded in freely flying bees (Baird et al., 2005). The error signal $\varepsilon_{F w d}$ (the input to the surge controller) is calculated as follows: 


$$
\varepsilon_{F w d}=\omega_{s e t F w d}-\max \left[\left(\omega_{R g h t}^{m}+\omega_{L e f t}^{m}\right),\left(\omega_{V t r l}^{m}+\omega_{D r s l}^{m}\right)\right]
$$

The surge controller was tuned using the same procedures as those previously described in the case of the LORA III autopilot (Serres et al., 2008a).

\subsection{Positioning control and positioning criterion}

The positioning control loop is in charge of positioning the bee with respect to either the side walls or the ground or the roof of the tunnel. Whether this positioning involves motion on the sway or the heave axis depends on whether the maximum OF measured is in the horizontal or vertical plane. The regulation process adopted here is based on the maximum value of the four OFs measured $\left(\max \left(\omega_{R g h t}^{m}, \omega_{\text {Left }}^{m}, \omega_{V t r l}^{m}, \omega_{D r s l}^{m}\right)\right.$, the red loop in Fig. 4), i.e., the value given by the nearest tunnel surface (walls, ground, or roof). This OF regulator is designed to maintain whichever of the four OFs measured is the larger equal to the positioning OF set-point $\omega_{\text {setPos }}$. The larger OF measured is compared with $\omega_{\text {setPos }}$, which was set at: $\omega_{\text {setPos }}=2.4 \mathrm{~V}$ (i.e., $315 \mathrm{deg} / \mathrm{sec}$ ). This value was again based on that recorded in freely flying bees (Baird et al., 2005). The error signal $\varepsilon_{\text {Pos }}$ (the input to the positioning controller) is calculated as follows:

$$
\varepsilon_{\text {Pos }}=\omega_{\text {setPos }}-\max \left(\omega_{R g h t}^{m}, \omega_{\text {Left }}^{m}, \omega_{V t r l}^{m}, \omega_{\text {Drsl }}^{m}\right)
$$

The positioning controller was tuned using the same procedures as those previously described in the case of the LORA III autopilot (Serres et al., 2008a).

(FIGURE 5about here) 
The surface that will be followed (walls, ground or roof) is specified by a Control direction Selector (Fig. 4, 5). The positioning control signal is multiplied by a direction factor that corresponds to the direction of the maximum OF signal. Note that the sway and heave dynamics can be driven alternately, depending on whichever (lateral or vertical) OF is maximum at any given time. The input to the type of dynamics is not commanded is then set at zero (Fig. 5) (Side thrust $=0$ or Vertical lift $=0$ ). The simulated bee will react to any unexpected changes in the OFs measured by adjusting either its lateral speed $V_{y}$ (and hence its lateral position) or its vertical speed $V_{z}$ (and hence its vertical position). The OF regulator will always react to the nearest of the four tunnel surfaces.

\section{SIMULATION RESULTS}

\subsection{Automatic tunnel-following}

In Fig. 6, the simulated environment is a straight tunnel 6 meters long, 1 meter wide, and 1 meter high. Fig. 6A shows a perspective view. Walls, ground, and roof were lined with natural grayscale images (Fig. 2). The simulated bee enters the tunnel at the speed $V_{x 0}=0.2 \mathrm{~m} / \mathrm{sec}$ and with the initial coordinates $x_{0}=0.1 \mathrm{~m}$, and various couples of $y_{0}$ and $z_{0}$ (Fig. 6B). Fig. $6 \mathrm{C}$ shows the five trajectories in the vertical plane $(\mathrm{x}, \mathrm{z})$ and Fig. $6 \mathrm{D}$ in the horizontal plane (x,y), plotted every $500 \mathrm{msec}$. Each bar indicates the honeybee's body orientation, which is known to form a fixed angle with the orientation of the mean flight-force vector (Nachtigall et al., 1971; David, 1978).

The simulated bee can be seen to have gradually increased both its height 
of flight (Fig. 6C) and its right clearance (Fig. 6D) to $0.33 \mathrm{~m}$, while the forward speed (Fig. 6E) increased automatically up to $2 \mathrm{~m} / \mathrm{sec}$ (i.e., the maximum speed allowed) whichever is the initial positions.

These results show that the ALIS autopilot caused the simulated bee to travel safely along the tunnel, while reaching a given forward speed and a given clearance from the walls.

\section{(FIGURE 6 and FIGURE 7 about here)}

\subsection{Effect of the local absence of contrast on one of the internal faces of the tunnel}

Fig. 7 shows successful tests on the behavior of the simulated bee in the presence of "no contrast" zones on the left wall or the roof of the tunnel. These "no contrast" zones could be either a real aperture or a lack of texture (Fig. 7A). The simulated bee was made to enter the tunnel at speed $V_{x 0}=$ $0.2 \mathrm{~m} / \mathrm{sec}$ with the initial coordinates $x_{0}=0.1 \mathrm{~m}, y_{0}=0.85 \mathrm{~m}, z_{0}=0.85 \mathrm{~m}$ (Fig. 7B). Fig. 7C shows the trajectory in the vertical plane (x, z) and Fig. 7D in the horizontal plane (x, y), plotted every 500 msec.

As can be seen from Fig. 7, the simulated bee was not greatly disturbed by either the 2-meter long aperture encountered on its left-hand side (at the beginning of the tunnel) or a similar aperture entering its dorsal field of view (at the end of the tunnel).

The positioning criterion (Fig. 7F) could select either the left or dorsal EMD output $\left(\omega_{\text {Left }}^{m}\right.$ or $\left.\omega_{D r s l}^{m}\right)$ when there were no lateral or vertical OF outputs because of the presence of "no contrast" zones (from $\mathrm{X}=0.5 \mathrm{~m}$ to $\mathrm{X}=2.5 \mathrm{~m}$ and from $\mathrm{X}=3.5 \mathrm{~m}$ to $\mathrm{X}=5.5 \mathrm{~m}$ ). The positioning criterion caused 
the simulated bee to keep a dorsal clearance $D_{\text {Drsl }}=0.35 \mathrm{~m}$ (Fig. $7 \mathrm{C}$ ) and a left clearance $D_{\text {Left }}=0.39 \mathrm{~m}$ (Fig. 7D) throughout its journey.

The forward criterion (Fig. 7G) could select either the vertical or horizontal EMD output when there were no lateral or vertical OF outputs because of the "no contrast" zones encountered (from $\mathrm{X}=0.5 \mathrm{~m}$ to $\mathrm{X}=2.5 \mathrm{~m}$ and from $\mathrm{X}=3.5 \mathrm{~m}$ to $\mathrm{X}=5.5 \mathrm{~m}$ ). This criterion caused the simulated bee to maintain a relatively constant speed $V_{x}=1.85 \mathrm{~m} / \mathrm{sec}$ throughout its journey (Fig. 7E).

These results show that the ALIS autopilot enabled the simulated bee to travel safely along the tunnel without being greatly disturbed by the presence of a lateral or dorsal "no contrast" zone.

\subsection{Automatic terrain-following}

Fig. 8 shows successful tests on the behaviour of the simulated bee on a sloping terrain (slope angle 7,deg). As this sloping zone gradually affected the relative distance from the bee to the ground $D_{V t r l}$, it acted like an OF perturbation (Fig. 8A). The simulated bee was made to enter the tunnel at the speed $V_{x 0}=0.2 \mathrm{~m} / \mathrm{sec}$ with the initial coordinates $x_{0}=0.1 \mathrm{~m}, y_{0}=$ $0.85 \mathrm{~m}, z_{0}=0.15 \mathrm{~m}$ (Fig. $8 \mathrm{~B}$ ). Fig. $8 \mathrm{C}$ shows the trajectory in the vertical plane (x, z) and Fig. 8D in the horizontal plane (x, y), plotted every $500 \mathrm{msec}$.

(FIGURE 8 about here)

As can be seen from Fig. 8, the simulated bee was not greatly disturbed by the ramp-like slope occurring below its flight path.

The positioning criterion (Fig. 8F) could select either the ventral or left EMD output $\left(\omega_{V t l r}^{m}\right.$ and $\left.\omega_{\text {Left }}^{m}\right)$. This automatic choice caused the simu- 
lated bee to maintain both a ventral clearance and a left clearance (Fig. 8D) throughout its journey.

The forward criterion can be seen to have mostly opted for vertical EMD outputs $\left(\omega_{V t l r}^{m}+\omega_{D r s l}^{m}\right.$, Fig. $\left.8 \mathrm{G}\right)$ because the ventral slope made the vertical section of the tunnel smaller than its horizontal section. This criterion caused the simulated bee to maintain a relatively constant speed $V_{x}=1.45 \mathrm{~m} / \mathrm{sec}$ throughout its journey (Fig. 8E).

These results show that the ALIS autopilot made the simulated bee travel along the tunnel without being greatly disturbed by the sloping ground encountered.

\subsection{Automatic speed control in horizontally and/or vertically tapered tunnels}

The simulated tunnels used here were 6-meter long, 1-meter high tapered tunnels with a 1-meter wide entrance and a 0.25-meter constriction halfway along the tunnel. This constriction could occur in either the horizontal plane (Fig. 9A) the vertical plane (Fig. 10A), or both planes together (Fig. 11A). These tunnels were designed to test the ability of the ALIS autopilot to overcome several strong OF disturbances at the same time.

\section{(FIGURE 9 and FIGURE 10 about here)}

As shown in Fig. 9, the simulated bee was made to enter a tunnel with a midway constriction in the horizontal plane, at the speed $V_{x 0}=0.2 \mathrm{~m} / \mathrm{sec}$ and with the initial coordinates $x_{0}=0.1 \mathrm{~m}, y_{0}=0.85 \mathrm{~m}, z_{0}=0.15 \mathrm{~m}$ (Fig. 9B). Fig. 9C shows the trajectory in the vertical plane (x, z) and Fig. 9D in the horizontal plane (x, y), plotted every 500 msec. 
The simulated bee followed the left wall of the tapered tunnel, simply because its starting point was close to that wall. The positioning criterion (Fig. 9F) selected the left EMD output $\left(\omega_{\text {left }}^{m}\right)$, which remained approximately equal to the positioning OF set-point throughout the journey (Fig. 9F). The simulated bee kept a safe left clearance throughout its journey. The simulated bee automatically slowed down as it approached the narrowest section of the tapered tunnel, and accelerated again when the tunnel widened out beyond that point (Fig. 9E). Since the tunnel narrowed only in the horizontal plane, the OF in the vertical plane was of little relevance to the speed control part of the ALIS autopilot. The forward speed depended mostly on the OF in the horizontal plane $\left(\omega_{\text {Left }}^{m}+\omega_{R g h t}^{m}\right.$, Fig. 9G) because the horizontal section of the tunnel was smaller than its vertical section.

The ALIS autopilot made the simulated bee travel safely along the "horizontal" tapered tunnel (tapering angle $7 \mathrm{deg}$ ) without being greatly perturbed by the major OF disturbance concomitantly detected by both its left and right OF sensors.

As shown in Fig. 10, the simulated bee was then made to enter a tunnel with a midway constriction in the vertical plane, at the speed $V_{x 0}=$ $0.2 \mathrm{~m} / \mathrm{sec}$, with the initial coordinates $x_{0}=0.1 \mathrm{~m}, y_{0}=0.85 \mathrm{~m}, z_{0}=0.15 \mathrm{~m}$ (Fig. 10B). Fig. 10C shows the trajectory in the vertical plane (x, $\mathrm{z})$ and Fig. 10D in the horizontal plane (x,y), plotted every 500 msec. In this case, the simulated bee followed both the ground and the left wall of the tapered tunnel, simply because its starting point was near to the ground and the left wall. The positioning criterion could select either the ventral or left OF measured ( $\omega_{V t l r}^{m}$ and $\left.\omega_{L e f t}^{m}\right)$, which remained approximately equal to the po- 
sitioning OF set-point throughout the journey (Fig. 10F). The simulated bee kept a safe ventral and left clearance throughout its journey.

The simulated bee automatically slowed down as it approached the narrowest section of the tapered tunnel, and accelerated again when the tunnel widened out beyond that point (Fig. 10E). As the tunnel narrowed only in the vertical plane, the $\mathrm{OF}$ in the horizontal plane was of little relevance to the speed control part of the ALIS autopilot. The forward speed depended mostly on the OF in the vertical plane $\left(\omega_{v t r l}^{m}+\omega_{\text {Drsl }}^{m}\right.$, Fig. 10G) because the vertical section of the tunnel was smaller than its horizontal section.

The ALIS autopilot made the simulated bee travel along the vertically tapered tunnel (tapering angle $7 \mathrm{deg}$ ) without being greatly perturbed by the major OF disturbance concomitantly detected by both its ventral and dorsal OF sensors.

\section{(FIGURE 11 about here)}

As shown in Fig. 11, the simulated bee was then made to enter the tunnel with midway constrictions in both the horizontal and vertical planes. The bee entered at the speed $V_{x 0}=0.2 \mathrm{~m} / \mathrm{sec}$ with the initial coordinates $x_{0}=0.1 \mathrm{~m}$, $y_{0}=0.85 \mathrm{~m}, z_{0}=0.15 \mathrm{~m}$ (Fig. 11B). Fig. $11 \mathrm{C}$ shows the trajectory in the vertical plane (x, z) and Fig. 11D in the horizontal plane (x, y), plotted every 500 msec. The simulated bee followed both the ground and the left wall of the tapered tunnel, simply because its starting point was near the ground and the left wall. The positioning criterion could select either the ventral or the left OF measured $\left(\omega_{V t r l}^{m}\right.$ and $\left.\omega_{\text {Left }}^{m}\right)$, which remained approximately equal to the positioning OF set-point throughout the trajectory (Fig. 11F). The simulated bee kept a safe ventral and left clearance throughout its journey. 
The simulated bee automatically slowed down as it approached the narrowest section of the tapered tunnel and accelerated again when the tunnel widened out beyond this point (Fig. 11E). As the tunnel narrowed in both the horizontal and vertical planes, the OFs in the horizontal and vertical planes were both equally relevant to the speed control part of the ALIS autopilot. The forward speed depended on the OFs in both the horizontal and vertical planes $\left(\omega_{R g h t}^{m}+\omega_{\text {Left }}^{m}\right.$ and $\omega_{V t l r}^{m}+\omega_{D r s l}^{m}$, Fig. 11G) because the horizontal and the vertical sections of the tunnel both varied to an equal extent.

The ALIS autopilot made the simulated bee cross the "horizontal and vertical" tapered tunnel (tapering angle $7 \mathrm{deg}$ in both planes) without being greatly perturbed by a major overall OF disturbance concomitantly affecting its lateral, ventral, and dorsal OF sensors.

All in all, these results show that the ALIS autopilot made the simulated bee:

- adopt a cruise speed that will automatically adjust to whichever section (horizontal or vertical) produces the largest optic flow, and

- adopt a clearance from one of the tunnel surfaces (the ground or the roof or one wall) that will be proportional to the animal's ground speed, thus automatically generating both terrain-following and wall-following behavior.

\section{CONCLUSIONS}

Here we have presented an OF-based 3D autopilot called ALIS. The results of the computer simulations described above show that a simulated bee 
equipped with the ALIS autopilot can navigate safely under purely visual control along a straight tunnel (Fig. 6), occurs even when part of the wall or the roof is devoid of texture (Fig. 7) and when the tunnel narrows or expands, in either the horizontal or vertical plane (Fig. 8A, 9A, 10A), or in both planes (Fig. 11A). Here we have not investigated dynamical disturbances such as wind perturbations but tested ALIS's robustness to strong OF perturbations. Absence of contrast on one side (as Fig. 7) and tapered tunnels (Fig. 9- 11) are considered by the ALIS control system (Fig. 4) as strong perturbations. The autopilot manages to cope with these major perturbations, allowing the simulated bee to fly safely in these tunnels.

These feats can all be achieved with a really minimalistic visual system consisting of only eight pixels forming four EMDs (two EMDs in the horizontal plane and two in the vertical plane). The ALIS autopilot enables the agent to avoid obstacles by performing maneuvers involving only translational DOFs (along $x, y, z$ ). The key to the performances of the ALIS autopilot is a pair of OF regulators designed to hold the perceived OF constant by adjusting the forward, side, and vertical thrusts. More specifically, these two OF regulators operate as follows:

(i) The first OF regulator adjusts the bee's forward speed so as to keep whichever sum of the two opposite OFs (i.e., left+right or ventral+dorsal) is maximum equal to a forward OF set-point. The outcome is that the bee's forward speed becomes proportional to the smallest dimension (width or height, or both) of the corridor (Fig. 9E, 10E, 11E). Further simulations showed (data not shown) that this occurs regardless of the position of the bee's starting point at the tunnel entrance. The forward speed attained by 
the simulated bee depends also on the forward OF set-point $\omega_{\text {setFwd }}$.

(ii) The second OF regulator adjusts the bee's lateral and vertical position so as to keep the largest OF value (from any of the four tunnel surfaces: walls, ground, or roof) equal to the positioning OF set-point. The outcome is that the clearance from the nearest wall (or ground or roof) becomes proportional to the bee's forward speed as defined in (i). The clearance from the nearest tunnel surface depends on the positioning OF set-point $\omega_{\text {setPos }}$.

The main advantage of this visuomotor control system is that it operates efficiently without any needs for explicit speed or distance information, and hence without any needs for speed or range sensors. The emphasis here is on behavior rather than metrics: the simulated bee behaves appropriately although it is completely "unaware" of its ground speed and its distance from the walls, ground, and roof. The simulated bee navigates on the basis of two parameters alone: the forward OF set-point $\omega_{\text {setFwd }}$ and the positioning OF set-point $\omega_{\text {setPos }}$ (Fig. 4). The explicit ALIS control scheme presented here (Fig. 4) can be viewed as a working hypothesis and is very much in line with the ecological approach (Gibson, 1950), according to which an animal's visual system is thought to drive the locomotor system directly, without requiring any "representation" of the environment (Franceschini et al., 1992; Duchon and Warren, 1994). The ALIS control scheme (Fig. 4) readily accounts for the behavior observed on real bees flying along a stationary corridor (Srinivasan et al., 1991; Serres et al., 2008b; Baird et al., 2006) or a tapered corridor (Srinivasan et al., 1996). It also accounts for the wall-following behavior observed in straight or tapered corridors (Serres et al., 2008b).

Real bees have 4500 ommatidia, per eye and obviously more than four 
OF sensors. These large number of OF sensors therefore enable them to measure the $\mathrm{OF}$ in many directions and an elaborated autopilot could make them to avoid obstacles occurring in many directions. An OF regulator is little demanding in terms of its neural (or electronic) implementation since it requires only a few linear operations (such as adding, subtracting, and applying various filters) and nonlinear operations (such as minimum and maximum detection). The minimalist control scheme described in this paper could be implemented in a micro-controller running at $1 \mathrm{kHz}$. In this way, the "computation time" could be up to $1 \mathrm{msec}$.

In terms of the potential applications of these findings, biomimetic solutions of the kind described here may pave the way for the design of computationlean, lightweight visual guidance systems for autonomous aerial, underwater, and space vehicles.

\section{ACKNOWLEDGMENTS}

We thank S. Viollet and L. Kerhuel for their fruitful comments and suggestions during this work, R. Brinkworth and D. O'Carroll (Adelaide Uni., Australia) for kindly making their panoramic images available to us, and J. Blanc for improving the English manuscript. This work was supported partly by CNRS (Life Science; Information and Engineering Science and Technology), by the Aix-Marseille University, by the French Defense Agency (DGA, 0534 022), by the French Agency for Research (ANR, RETINAE project), and by the European Space Agency (ESA) under contract n 08-6303b. 


\section{References}

Altshuler, D.L., Dickson, W.B., Vance, J.T., Roberts, S.P., and Dickinson, M.H. (2005). Short-amplitude high-frequency wing strokes determine the aerodynamics of honeybee flight. PNAS. 102(50), 821318218.

Aubépart, F., and Franceschini, N. (2007). A bio-inspired optic flow sensor based on FPGA: application to micro-air-vehicles. J. of Microprocessors and Microsystems. 31(6), 408-419.

Baird, E., Srinivasan, M.V., Zhang, S., and Cowling, A. (2005). Visual control of flight speed in honeybees. J. Exp. Biol. 208, 3895-3905.

Baird, E., Srinivasan, M.V., Zhang, S., Lamont, R., and Cowling, A. (2006). Visual control of flight speed and height in honeybee. LNAI. 4095, 40-51.

Beyeler, A., Zufferey, J.-C., and Floreano, D. (2007). 3D vision-based navigation for indoor microflyers. Proc. IEEE Int. Conf. on Robotics and Automation. pp. 1336-1341.

Blanes, C. (1986). Appareil visuel élémentaire pour la navigation à vue d'un robot mobile autonome. MS in Neuroscience, Marseille, France: University of Aix-Marseille II.

Brinkworth, R.S.A., and O'Carroll, D.C. (2007). Biomimetic Motion Detection. Proc. of the Int. Conf. on Intelligent Sensors, Sensor Networks and Information Processing (ISSNIP). 3, 137-142.

Coombs, D., and Roberts, K. (1992). Bee-bot: using peripheral optical flow to avoid obstacle. SPIE: Intelligent robots and computer vision XI. 1825, 714-721.

David, C. (1978). The relationship between body angle and flight speed in free-flying Drosophila. Physiol. Entomol. 3, 191-195.

Dickson, W.B., Straw, A.D., Poelma, C., and Dickinson, M.H. (2006). An integrative model of insect flight control. Proc. of the 44th AIAA Aerospace Sciences Meeting and Exhibit. AIAA-2006-0034.

Dillon, M.E., and Dudley, R. (2004). Allometry of maximum vertical force production during hovering flight of neotropical orchid bees (Apidae: Euglossini). J. Exp. Biol. 207, 417-425.

Duchon, A.P., and Warren, W.H. (1994). Robot navigation from a Gibsonian viewpoint. Proc. Int. Conf. Syst. Man and Cyb. 2272-2277, San Antonio, Texas.

Dudley, R. (1995). Extraordinary flight performance of orchid bees (apidae: euglossini) hovering in heliox (80\% He / 20\% O²). J. Exp. Biol. 198, 1065-1070. 
Dudley, R. (2000). The biomechanics of insect flight: form, function, evolution, (Princeton: Princeton University Press).

Ellington, C.P. (1984). The aerodynamics of hovering insect flight. III. Kinematics. Phil. Trans. Roy. Soc. Lond. B. 305, 41-78.

Esch, H., Nachtigall, W., and Kogge, S.N. (1975). Correlations between aerodynamic output, electrical activity in the indirect flight muscles and wing positions of bees flying in a servomechanically controlled flight tunnel. J. Comp. Physiol. 100, 147-159.

Franceschini, N., Pichon, J.M., and Blanes, C. (1992). From insect vision to robot vision. Philos. Trans. R. Soc. Lond. B 337, 283294.

Franceschini, N., Ruffier, F., and Serres, J. (2007). A bio-inspired flying robot sheds light on insect piloting abilities. Current Biology. 17, 329-335.

Franceschini, N., Ruffier, F., Serres, J., and Viollet, S. (2009). Optic flow based visual guidance: from flying insects to miniature aerial vehicules. In Aerial Vehicles, T.M. Lam, ed. (In-Tech), pp. 747-770.

von Frisch, K. (1948). Gelöste und ungelöste Rätsel der Bienensprache. Naturwissenschaften. 35, 38-43.

Gibson, J.J. (1950). The perception of the visual world. (Boston: Houghton Mifflin).

Horridge, G.A. (1987). The evolution of visual processing and the construction of seeing system. Proc. Roy. Soc. Lond. B. 230, 279-292.

Humbert, J.S., Hyslop, A., and Chinn, M. (2007). Experimental validation of wide-field integration methods for autonomous navigation. Proc. IEEE int. conf. intelligent robots and systems.

Ibbotson, M.R. (2001). Evidence for velocity-tuned motion-sentive descending neurons in the honeybee. Proc. Roy. Soc. Lond. B. 268, 2195-2201.

Lewis, M.A. (1997). Visual Navigation in a Robot using Zig-Zag Behavior, NIPS.

Nachtigall, W., Widmann, R., and Renner, M. (1971). Uber den ortsfesten freien Flug von Bienen in einem Saugkanal. Apidologie. 2, 271-282.

Netter, T., and Franceschini, N. (2002). A robotic aircraft that follows terrain using a neuromorphic eye. Proc. IEEE int. conf. intelligent robots and systems. 129-134.

Neumann, T.R., and Bülthoff, H.H. (2001). Insect visual control of translatory flight. LNCS/LNAI. 2159, 627-636. 
Pichon, J.M., Blanes, C., and Franceschini, N. (1989). Visual guidance of a mobile robot equipped with a network of self-motion sensors. Proc. SPIE 1195, 44-53.

Preiss, R. (1987). Motion parallax and figural properties of depth control flight speed in an insect. Biol. Cyb. 57, 1-9.

Pudas, M., et al. (2007). A miniature bio-inspired optic flow sensor based on low temperature co-fired ceramics (LTCC) technology. Sensors and Actuators A. 133, 88-95.

Riley, J.R., et al. (2003). The Automatic Pilot of Honeybees. Proc. Roy. Soc. Lond. 270, 2421-2414.

Ruffier, F., and Franceschini, N. (2003). Bio-inspired optical flow circuits for the visual guidance of microair vehicles. IEEE int. symp. circuits and systems. 3, 846-849.

Ruffier, F., and Franceschini, N. (2005). Optic flow regulation: the key to aircraft automatic guidance. Robotics and Autonomous Systems. 50(4), 177-194.

Sane, S.P., and Dickinson, M.H. (2002). The aerodynamic effects of wing rotation and a revised quasisteady model of flapping flight. J. Exp. Biol. 205, 1087-1096.

Santos-Victor, J., Sandini, G., Curotto, F., and Garibaldi, S. (1995). Divergent stereo in autonomous navigation: from bees to robots. Int. J. of Comp. Vision. 14, 159-177.

Schilstra. C., and van Hateren, J.H. (1999). Blowfly flight and optic flow. I. Thorax kinematics and flight dynamics. J. Exp. Biol. 202, 1481-1490.

Seidl, R., and Kaiser, W. (1981). Visual field size, binocular domain and the ommatidial array of the compound eyes in worker honey bees. J. Comp. Physiol. A 143, 1726.

Serres, J., Dray, D., Ruffier, F., and Franceschini, N. (2008). A vision-based autopilot for a miniature air-vehicle: joint speed control and lateral obstacle avoidance. Autonomous Robot. 25, 103 -122.

Serres, J., Ruffier, F., Masson, G.P., and Franceschini, N. (2008). A bee in the corridor: centering and wall-following. Naturwissenschaften. 95, 1181-1187.

Srinivasan, M.V., Lehrer, M., Kirchner, W.H., and Zhang, S.W. (1991). Range perception through apparent image speed in freely flying honeybees. Vis. Neurosci. 6, 519-535.

Srinivasan, M.V., Zhang, S.W., Lehrer, M., and Collett, T.S. (1996). Honeybee navigation en route to the goal: visual flight control and odometry. J. Exp. Biol. 199, 237-2446.

Srinivasan, M.V., Zhang, S.W., Chahl, J.S., Barth, E., and Venkatesh, S. (2000). How honeybees make grazing landings on flat surfaces. Biol. Cyb. 83, 171-183. 
Tammero, L.F., and Dickinson, M.H. (2002). Collision-avoidance and landing responses are mediated by separate pathways in the fruit fly, Drosophila melanogaster. J. Exp. Biol. 205, 2785-2798.

Vickers, N.J, and Baker, T.C. (1994). Visual feedback in the control of pheromone-mediated flight in Heliothis virescens males (Lepidoptera: noctuidae). J. Insct. Behav. 7, 605-631.

Wakakuwa, M., Kurasawa, M., Giurfa, M., and Arikawa, K. (2005). Spectral heterogeneity of honeybee ommatidia. Naturw. 92, 464-467.

Weber, K., Venkatesh, S., and Srinivasan, M.V. (1997). Insect inspired behaviours for the autonomous control of mobile robots, M.V. Srinivasan and S. Venkatesh, Eds. From living eyes to seeing machines. (Oxford: Oxford University Press).

Zeil, J., Boeddeker, N., and Hemmi, J.M. (2008) Visualy guided behavior. In New Encyclopedia of Neuroscience (Amsterdam: Elsevier Science Publishers), in press, 2008.

Zufferey, J.-C., and Floreano, D. (2005). Toward 30-gram autonomous indoor aircraft: vision-based obstacle avoidance and altitude control. Proc. IEEE Int. Conf. on Robotics and Automation. pp. 2594-2599, Barcelona, Spain. 


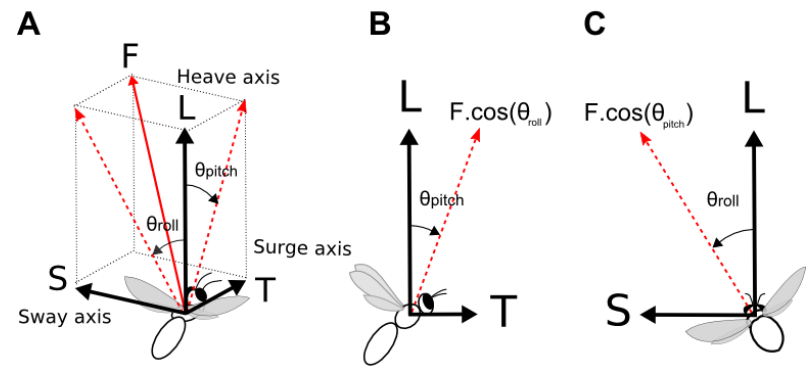

Figure 1: (A) Resolution of the mean flight-force vector $\vec{F}$ along the surge $\mathrm{X}$-axis giving the forward thrust $T$, along the sway $\mathrm{Y}$-axis giving the side thrust $S$, and along the heave $\mathrm{Z}$-axis giving the vertical lift $L$. (B) Pitching the mean flight-force vector $\vec{F}$ by an angle $\theta_{\text {pitch }}$ generates a forward thrust $T$. (C) Rolling the mean flight-force vector $\vec{F}$ by an angle $\theta_{\text {roll }}$ generates a side thrust $S$.
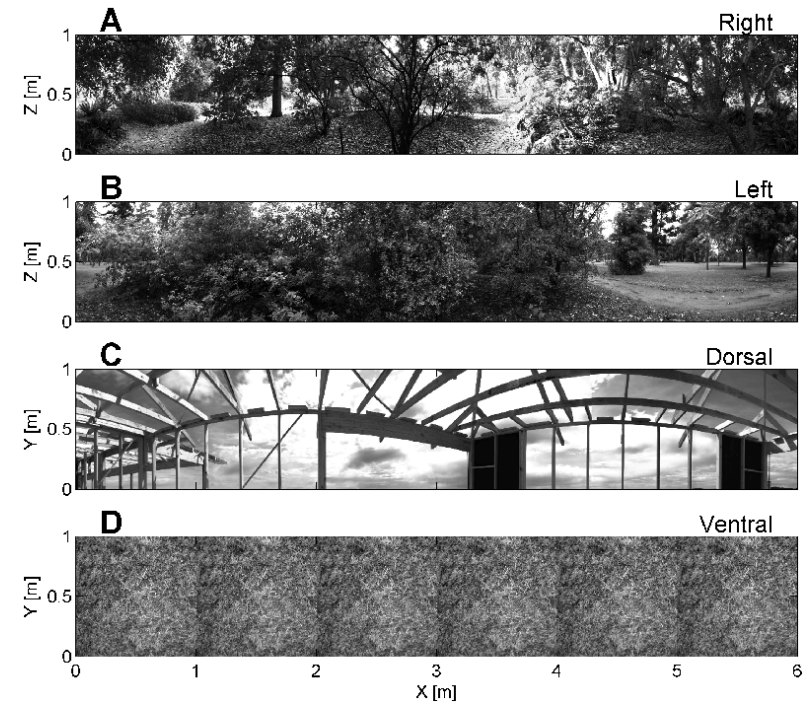

Figure 2: The grayscale natural scenes used to line the 4 internal faces of the simulated tunnel. Resolution of the images was $1000 \times 6000$ pixels $\left(1 \mathrm{pixel}=1 \mathrm{~mm}^{2}\right)$. Images are therefore $1 \times 6$-meter in size. All four faces of the tunnel were lined with different images: right wall (A), left wall $(B)$, roof $(\mathrm{C})$, and ground (D). 
A

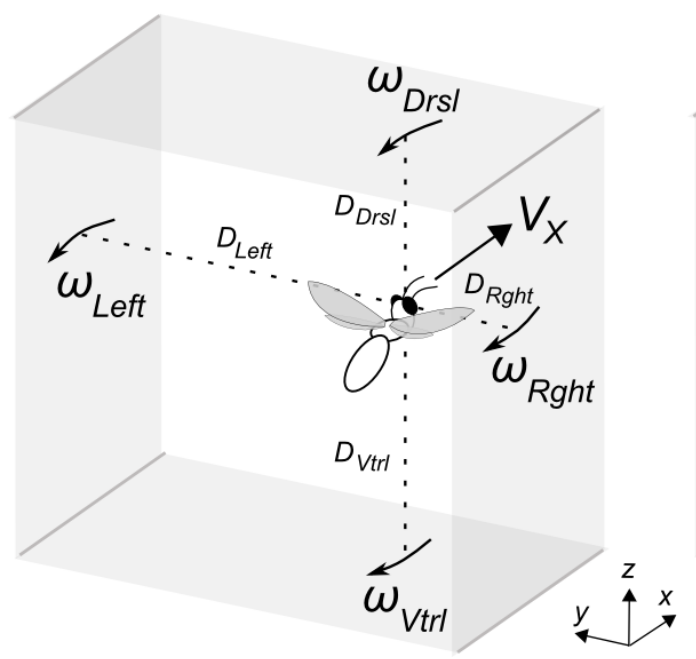

B

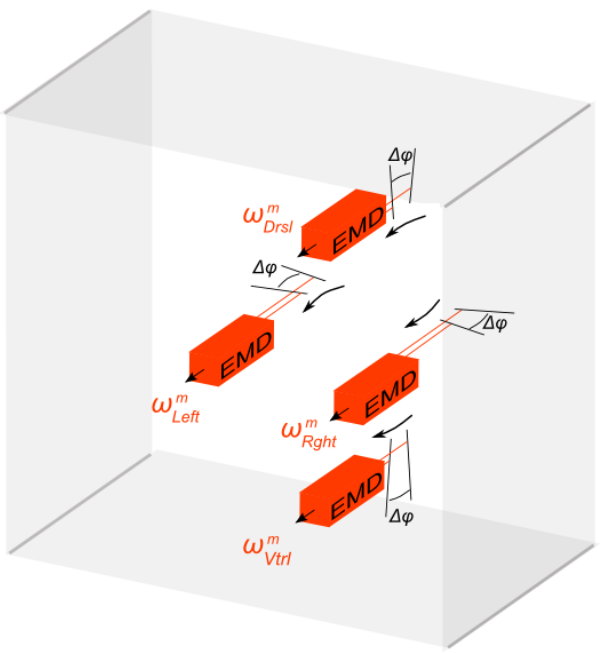

Figure 3: (A) A simulated bee flying at forward speed $V_{x}$ along a tunnel generates an OF (Eq. 8) that depends on the perpendicular distance (right $D_{R g h t}$, left $D_{L e f t}$, ventral $D_{V t r l}$, dorsal $D_{D r s l}$ ) from the tunnel surfaces. The simulated bee is equipped with four OF sensors. The sensors'axes are always oriented at fixed roll and pitch orientations, perpendicular to the walls, ground and roof, respectively, and the OF is generated laterally $\left(\omega_{L e f t}\right.$ and $\left.\omega_{R g h t}\right)$, ventrally $\left(\omega_{V t r l}\right)$ and dorsally $\left(\omega_{D r s l}\right)$. (B) Each OF sensor consists of only two photoreceptors (two pixels) driving an Elementary Motion Detector (EMD). The visual axes of the two photoreceptors are separated by an interreceptor angle $\Delta \varphi=4 \mathrm{deg}$. 


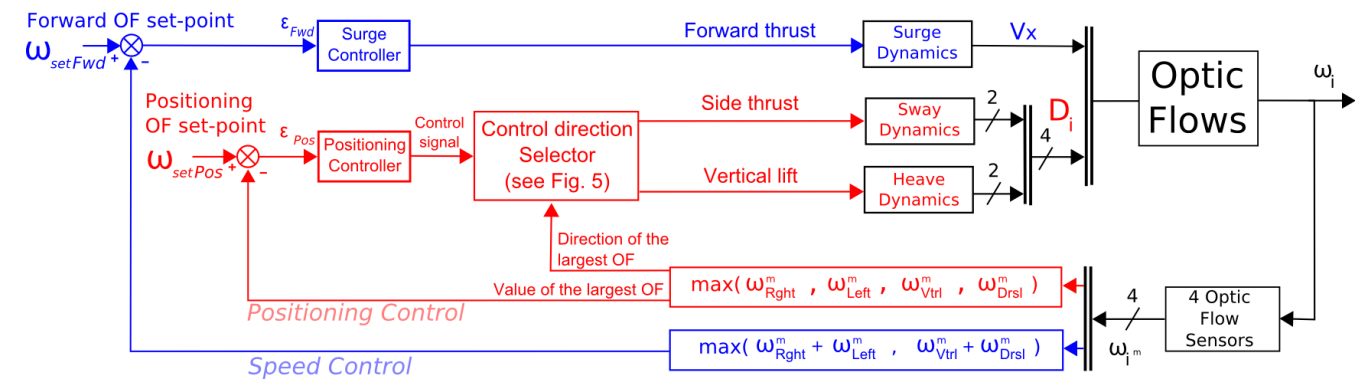

Figure 4: The ALIS autopilot is based on two interdependent visual feedback loops, each with its own OF set-point: a speed control loop (in blue) and a positioning control loop (in red). The surge controller adjusts the pitch angle $\theta_{\text {pitch }}$ (that determines $V_{x}$ via the bees' surge dynamics) on the basis of whichever sum of the two coplanar (horizontal or vertical) OFs measured is the largest. This value is compared with the forward OF set-point $\omega_{\text {setFwd }}$. The surge controller commands the forward speed so as to minimize the error $\varepsilon_{F w d}$. The positioning controller controls the roll angle $\theta_{\text {roll }}$ (or the stroke amplitude $\Delta \Phi$ ), which determines the distances to the walls (or the distances to the ground and to the roof), depending on the sway (or heave) dynamics on the basis of whichever of the four measured OFs is the largest. The latter

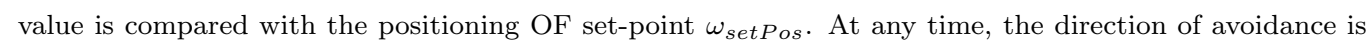
given by a Control direction Selector that multiplies the control signal by a direction factor depending on the direction of the maximum OF signal (see Fig. 5). The positioning controller (Proportional-Derivative, PD) commands the sway (or heave) dynamics so as to minimize the error $\varepsilon_{P o s}$. The dash accross the connection lines indicates the number of variables involved. $D_{i}$ is the distance to the surface involved (see Eq. 7). 


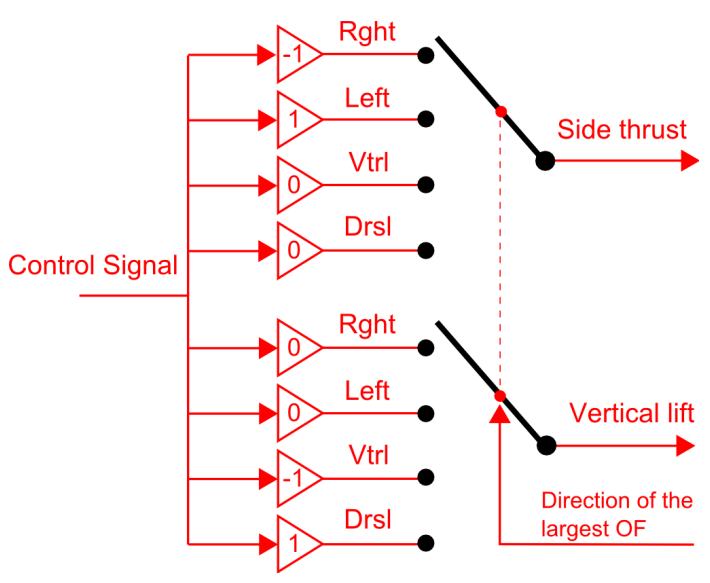

Figure 5: The Control direction Selector automatically selects the tunnel surface to be followed (wall, ground or roof) by multiplying the control signal (the output from the Positioning controller) by a direction factor that depends on the direction of the largest $O F$ signal. Note that the sway and heave dynamics can be driven alternately, depending on which OF (side or vertical) is the largest at any given time. The input to the sway or heave dynamics that is not relevant is set to zero. In the example shown here, the direction of the maximum OF is "right". Consequently, the output for the Side thrust is the control signal multiplied by -1 and the output for the Vertical thrust is the control signal multiplied by 0 . 

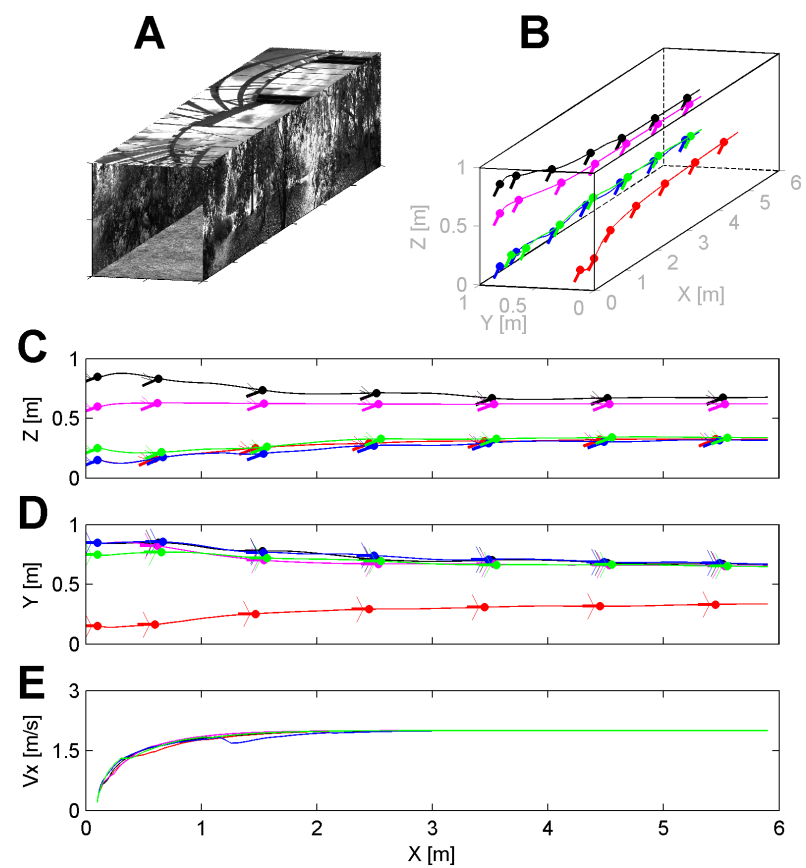

Figure 6: (A) Perspective view of the straight flight tunnel. (B) Simulated bee's 3-D trajectory starting at $x_{0}=0.1 \mathrm{~m}$, with initial speed $V_{x o}=0.2 \mathrm{~m} / \mathrm{sec}$, and various $y_{0}$ and $z_{0}$, plotted every $500 \mathrm{msec}$. (C) Trajectory in the vertical plane (x, z), every $500 \mathrm{msec}$. (D) Flight track in the horizontal plane (x, y), plotted every 500 msec. (E) Forward speed $V_{x}$ profile. 

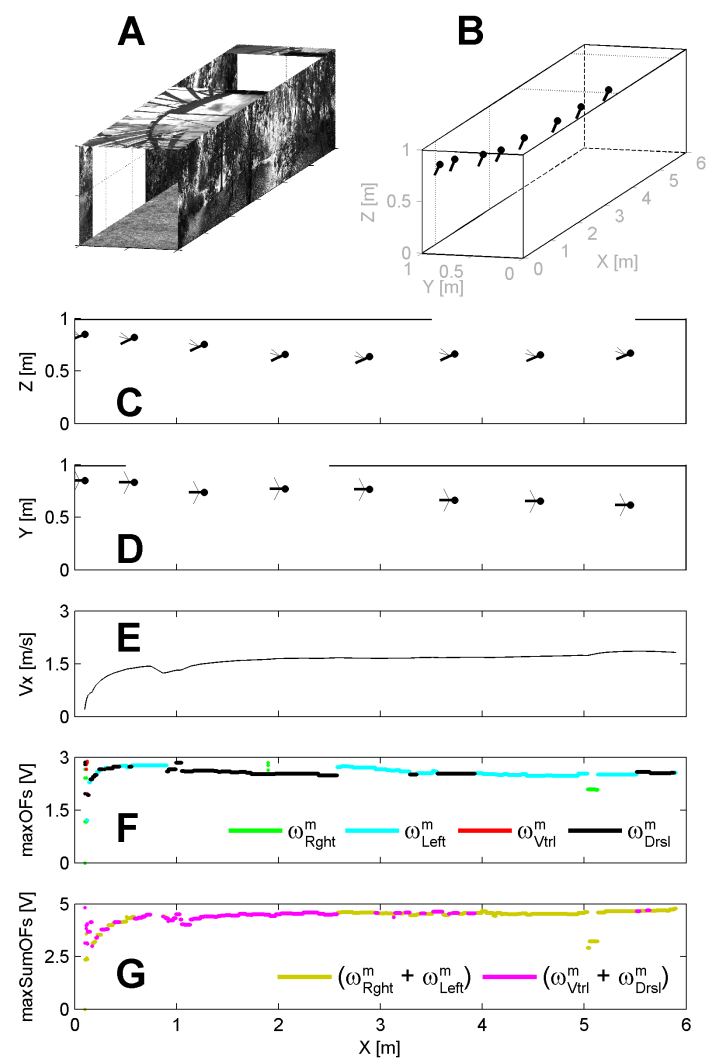

Figure 7: (A) Perspective view of the straight flight tunnel including two "no contrast" zones. (B)Simulated bee's 3-D trajectory starting at $x_{0}=0.1 \mathrm{~m}, y_{0}=0.85 \mathrm{~m}, z_{0}=0.85 \mathrm{~m}$, at the forward speed $V_{x o}=0.2 \mathrm{~m} / \mathrm{sec}$, plotted every $500 \mathrm{msec}$. (C) Trajectory in the vertical plane (x, z), every $500 \mathrm{msec}$. (D) Trajectory in the horizontal plane (x,y), plotted every $500 \mathrm{msec}$. (E) Forward speed $V_{x}$ profile. (F) Positioning feedback signal determined by the largest output from the four OF sensors (right OF sensor = green; left OF sensor = cyan; ventral OF sensor = red; dorsal OF sensor = black). (G) Forward feedback signal determined by the largest sum of the two diametrically opposed OF sensors (horizontal OF sensors = yellow; vertical OF sensors = magenta). 

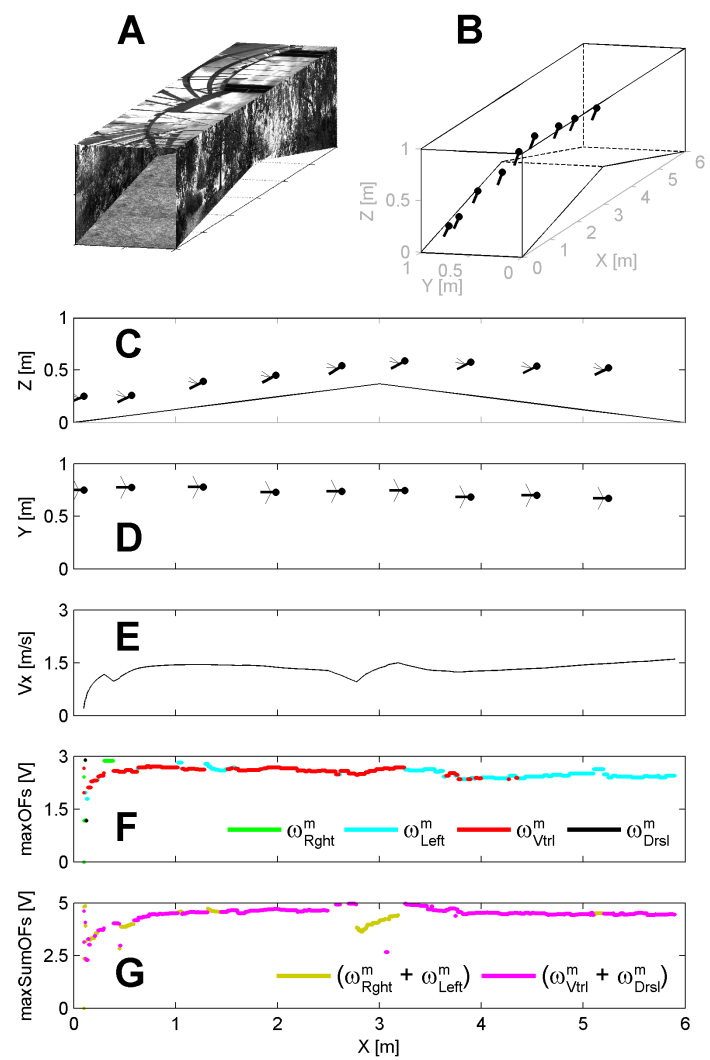

Figure 8: (A) Perspective view of the tapered tunnel. (B) Simulated bee's 3-D trajectory starting at the initial coordinates $x_{0}=0.1 \mathrm{~m}, y_{0}=0.75 \mathrm{~m}, z_{0}=0.25 \mathrm{~m}$, and at the speed $V_{x o}=0.2 \mathrm{~m} / \mathrm{sec}$, plotted every 500 msec. (C) Trajectory in the vertical plane (x, z), every 500 msec. (D) Trajectory in the horizontal plane (x, y), plotted every 500 msec. (E) Forward speed $V_{x}$ profile. (F) Positioning feedback signal determined by the largest output from the four OF sensors (right OF sensor = green; left OF sensor = cyan; ventral OF sensor = red; dorsal OF sensor = black). (G) Forward feedback signal determined by the largest sum of the two diametrically opposed OF sensors (horizontal OF sensors = yellow; vertical OF sensors = magenta). 

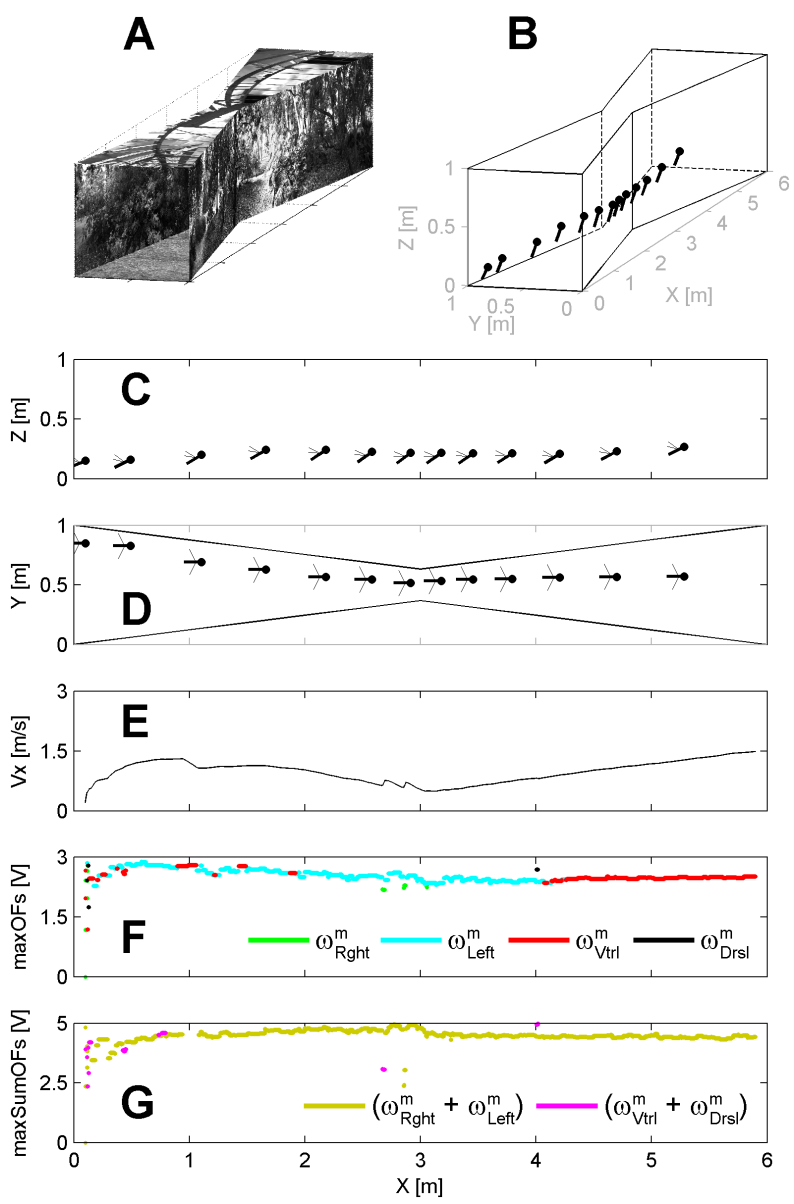

Figure 9: (A) Perspective view of the tapered tunnel. (B) Simulated bee's 3-D trajectory starting at the initial coordinates $x_{0}=0.1 \mathrm{~m}, y_{0}=0.85 \mathrm{~m}, z_{0}=0.15 \mathrm{~m}$, and at the speed $V_{x o}=0.2 \mathrm{~m} / \mathrm{sec}$, plotted every 500 msec. (C) Trajectory in the vertical plane (x, z), every $500 \mathrm{msec}$. (D) Trajectory in the horizontal plane (x, y), plotted every 500 msec. (E) Forward speed $V_{x}$ profile. (F) Positioning feedback signal determined by the largest output from the four OF sensors (right OF sensor = green; left OF sensor = cyan; ventral OF sensor $=$ red; dorsal OF sensor = black). (G) Forward feedback signal determined by the largest sum of the two diametrically opposed OF sensors (horizontal OF sensors = yellow; vertical OF sensors $=$ magenta). 

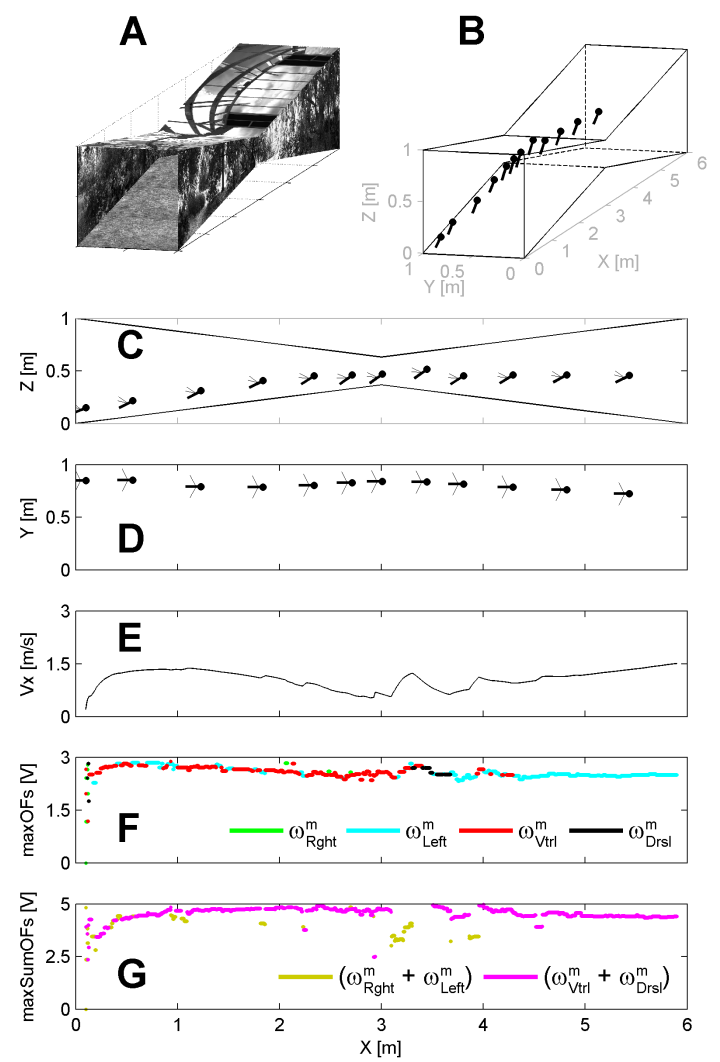

Figure 10: (A) Perspective view of the tapered tunnel. (B) Simulated bee's 3-D trajectory starting at the initial coordinates $x_{0}=0.1 \mathrm{~m}, y_{0}=0.85 \mathrm{~m}, z_{0}=0.15 \mathrm{~m}$, and at the speed $V_{x o}=0.2 \mathrm{~m} / \mathrm{sec}$, plotted every $500 \mathrm{msec}$ (C) Trajectory in the vertical plane (x, z), every $500 \mathrm{msec}$. (D) Trajectory in the horizontal plane (x, y), plotted every $500 \mathrm{msec}$. (E) Forward speed $V_{x}$ profile. (F) Positioning feedback signal determined by the largest output from the four OF sensors (right OF sensor = green; left OF sensor = cyan; ventral OF sensor = red; dorsal OF sensor = black). (G) Forward feedback signal determined by the largest sum of the two diametrically opposed OF sensors (horizontal OF sensors = yellow; vertical OF sensors $=$ magenta) . 

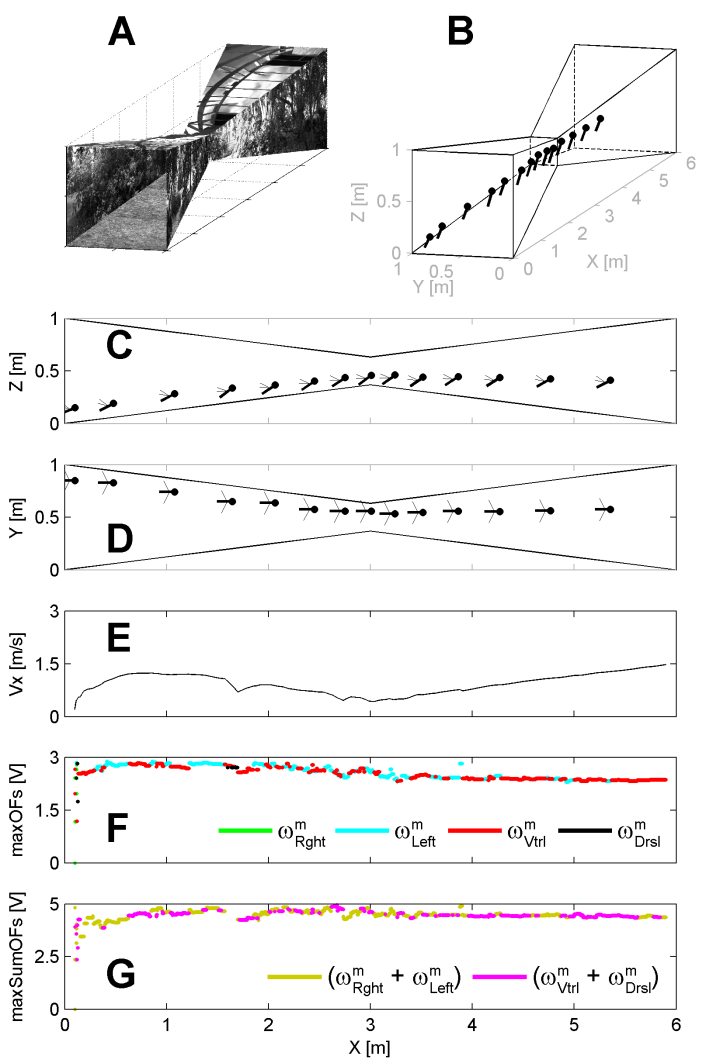

Figure 11: (A) Perspective view of the tapered tunnel. (B) Simulated bee's 3-D trajectory starting at initial coordinates $x_{0}=0.1 \mathrm{~m}, y_{0}=0.85 \mathrm{~m}, z_{0}=0.15 \mathrm{~m}$, and at the speed $V_{x o}=0.2 \mathrm{~m} / \mathrm{sec}$, plotted every 500 msec. (C) Trajectory in the vertical plane (x, z), every 500 msec. (D) Trajectory in the horizontal plane (x, y), plotted every 500 msec. (E) Forward speed $V_{x}$ profile. (F) Positioning feedback signal determined by the largest output from the four OF sensors (right OF sensor = green; left OF sensor = cyan; ventral OF sensor $=$ red; dorsal OF sensor = black). (G) Forward feedback signal determined by the largest sum of the two diametrically opposed OF sensors (horizontal OF sensors = yellow; vertical OF sensors = magenta). 